\title{
La exactitud posicional de la cartografía histórica
}

Gonzalo Moreno Vergara, Joaquín Cortés José, Instituto de Cartografía de Andalucía

\section{INTRODUCCIÓN}

El cartógrafo, al realizar un simple croquis o la cartografía más precisa, está representando la realidad al situar sobre el plano una serie de elementos territoriales que están relacionados, por las distancias y los ángulos que los separan, por la continuidad o adyacencia, etc., pero sólo cuando se establece una razón de proporcionalidad (la escala) entre el territorio y su representación se puede hablar de mapa.

El propósito de este texto es comentar el método de análisis de la evolución de la precisión de las técnicas de posicionamiento en la cartografía antigua, que se ha empleado para explicar la construcción de la imagen cartográfica de Andalucia del siglo XVI al XIX, uno de los capitulos de la obra Andalucía, la imagen cartográfica hasta fines del siglo XIX.

\section{ANÁLISIS DE LA ESCALA}

Una primera aproximación en el estudio de un mapa antiguo es a través del análisis de su escala. Habitualmente viene establecida mediante una expresión literal, por ejemplo, "20 leguas comunes de 171/2 al grado", y una escala gráfica o "troncos de leguas" donde se representa la equivalencia de las medidas en papel con las distancias en el terreno. Una de las primeras dificultades estriba en determinar qué tipo de unidades antiguas se está empleando (generalmente leguas o millas) y, dentro de cada una de ellas, sus magnitudes que variaban según la época y el lugar.

Las marcas de latitud y longitud también permiten deducir la escala de un mapa, por lo que conviene verificar la coherencia entre los valores de escala determinados por la medida de un grado de meridiano y uno de paralelo con las del tronco de leguas. Por último, otra manera de calcular la escala es mediante el cociente entre la distancia que separa a dos elementos sobre el mapa y la correspondiente en la realidad. Este cociente estará afectado de los propios errores presentes en el mapa, por lo que se debería repetir la operación utilizando diferentes pares de puntos y finalmente tomar el valor medio. La escala asi determinada es más correcta desde el punto de vista del usuario pues realmente relaciona las medidas sobre el mapa con la realidad. Además, la dispersión en los valores de los cocientes es indicativa de los errores aleatorios en la posición de los elementos y del nivel de homogeneidad en la escala.

\begin{tabular}{lllll} 
& & $\begin{array}{c}\text { MAPA } \\
\text { [METROS] }\end{array}$ & $\begin{array}{c}\text { ReAlidAD } \\
\text { [METROS }\end{array}$ & Cociente \\
Cádiz & Córdoba & 0,1850 & 202.356 & 1.093 .816 \\
Cádiz & Gibraltar & 0,0860 & 98.192 & 1.141 .767 \\
Cádiz & Jaén & 0,2575 & 260.619 & 1.012 .113 \\
Cádiz & Málaga & 0,1579 & 170.265 & 1.078 .480 \\
Cádiz & Sevilla & 0,1038 & 99.754 & 961.484 \\
(...) & & & & \\
Jaén & Málaga & 0,1388 & 127.173 & 916.562 \\
Jaén & Sevilla & 0,1934 & 195.516 & 1.011 .072 \\
Málaga & Sevilla & 0,1553 & 156.382 & 1.007 .291 \\
& & & media & 1.038 .083 \\
& & & mediana & 1.020 .175 \\
\hline
\end{tabular}

Escala por relación de distancias realidad/mapa (J. Hondius, 1606)

\section{GEORREFERENCIACIÓN}

La georreferenciación permite analizar el posicionamiento de los elementos presentes en un mapa. El procedimiento a seguir depende de la información disponible en el mismo. Variará según se disponga o no de marcas de latitud/longitud o líneas con los meridianos y paralelos que permitan determinar las coordenadas geográficas de cualquier punto y estudiar la exactitud posicional absoluta. En caso de no disponer de dichas marcas de referencia, tan sólo se podrá analizar la exactitud relativa entre las posiciones de los elementos.

- Absoluta.

El primer paso es determinar el sistema de proyección en el que se encuentra el mapa. El objetivo es poder aplicar una transformación polinómica de primer grado, una conforme o una afín, sin necesidad de deformar la hoja para adaptarla a otra proyección. Una vez georreferenciado, se puede reproyectar al sistema que se quiera (por ejemplo, a UTM) para compararlo con la cartografía actual.

Generalmente los mapas antiguos no suelen indicar la proyección empleada. No obstante, la propia malla de paralelos y me- 
ridianos ayuda a deducir qué tipo de sistema se está utilizando. Por ejemplo, si los meridianos son rectos y paralelos, se tratará de una cilindrica directa (tal vez una Mercator); si los meridianos convergen y los paralelos son curvos, lo normal es que sea una cónica; etc. Aspectos como el espaciado entre las líneas, su curvatura o el ángulo con el que se cortan permiten afinar en la determinación de la proyección. La extensión del territorio abarcado también puede ayudar, porque cuanto mayor sea, más fácil es deducir la proyección, aunque también será más relevante elegir la correcta.

Cuando no se puede saber la proyección o bien el mapa original o la copia con la que se trabaja presentan deformaciones, se hace necesario utilizar polinomios de segundo o tercer grado para absorber estas deficiencias y adaptar la malla de la carta a la de una proyección conocida; en este caso se debe emplear un mayor número de puntos de control distribuidos por todo el mapa para determinar unos parámetros de transformación adecuados.

Por otro lado, un aspecto fundamental para una correcta georreferenciación es la determinación del meridiano origen al que se refieren las longitudes. En algunos casos viene indicado expresamente en la carta, pero no siempre ocurre así. Una forma de hallarlo es restar a la longitud de uno o varios puntos significativos (p. ej., ciudades) la que le corresponde actualmente referida a Greenwich. Esta diferencia será aproximadamente la longitud (con respecto a Greenwich) del meridiano origen del mapa. No es necesario extenderse en decir que cuando se produce un error en este sentido hay un desplazamiento del mapa en dirección este-oeste.

\section{- Relativa.}

No cabe duda del interés de poder realizar un posicionamiento absoluto de los mapas. Sin embargo, en algunos de ellos no se dispone de referencia alguna a coordenadas geográficas referidas a la Tierra. No obstante, un usuario convencional lo que requiere habitualmente es conocer la posición de unos elementos con respecto a otros, y poder determinar distancias y ángulos entre ellos, por lo que es importante analizar la exactitud relativa que presentan.

La georreferenciación de estos mapas se realiza fijando la posición de algunos elementos significativos con la de sus homólogos en la realidad. Conviene utilizar sólo polinomios de primer grado, es decir, transformaciones de 4 ó 6 parámetros (traslación, giro y escalado), ya que los propios puntos de apoyo poseen errores y el empleo de polinomios de mayor grado tan sólo conseguiría absorber mejor estos errores en el entorno de los puntos e introducir deformaciones en el resto del mapa. Si se dispone de tronco de leguas con el que determinar la escala, se puede fijar este parámetro y dejar libres sólo la traslación y el giro.

Es interesante que el ajuste se realice por mínimos cuadrados empleando todos los puntos disponibles. De esta forma se minimizan los residuos al cuadrado y éstos son significativos del error en la posición de cada punto con respecto al resto, es decir, dan una medida de la exactitud relativa

Ante la imposibilidad de conocer la proyección, el planteamiento más lógico es utilizar una conforme ya que en ellas se conservan las formas de los elementos y las distancias relativas a nivel local, aunque en áreas más extensas se van apreciando diferencias en las formas y cambios en el factor de escala que dependerán de la proyección. En un territorio como Andalucia, estas discrepancias llegan a ser notables, aunque su repercusión dependerá del nivel de exactitud del mapa bajo estudio.

- Reajustes.

En ocasiones, en mapas georreferenciados en modo absoluto, se detectan errores sistemáticos importantes (desplazamientos y escalados principalmente). En estos casos, resulta conveniente realizar una nueva transformación que trate de eliminar los sistematismos y ajustar los elementos cartografiados a su posición actual, dejando sólo los errores aleatorios. Una forma de reajustar es aplicar la misma metodología descrita en la georreferenciación en modo relativo, es decir, utilizando elementos identificables en la cartografía y sus homólogos en la realidad.

Otra posibilidad es determinar el patrón que siguen los errores sistemáticos y aplicar las correcciones necesarias para contrarrestarlos. Esto permite, además, arrojar luz sobre errores conceptuales que se podian estar cometiendo. Un ejemplo se encuentra en el mapa Andaluzia nova descript de J. Hondius, fechado en 1606. En él se observa un desplazamiento importante hacia el este y un tamaño notablemente superior a la realidad.

Efectivamente, al comparar la escala determinada por la medida de un grado de meridiano (1:1.357.100) con la calculada a partir de las distancias entre ciudades (1:1.035.300), se obtuvo un cociente de 1,31. Teniendo presente por otra documentación que el meridiano origen debía de ser el pico Teide $\left(16^{\circ} 35^{\prime}\right.$ al oeste de Greenwich) y tomando como paralelo central el de $36^{\circ} 30^{\prime}$, se apli-

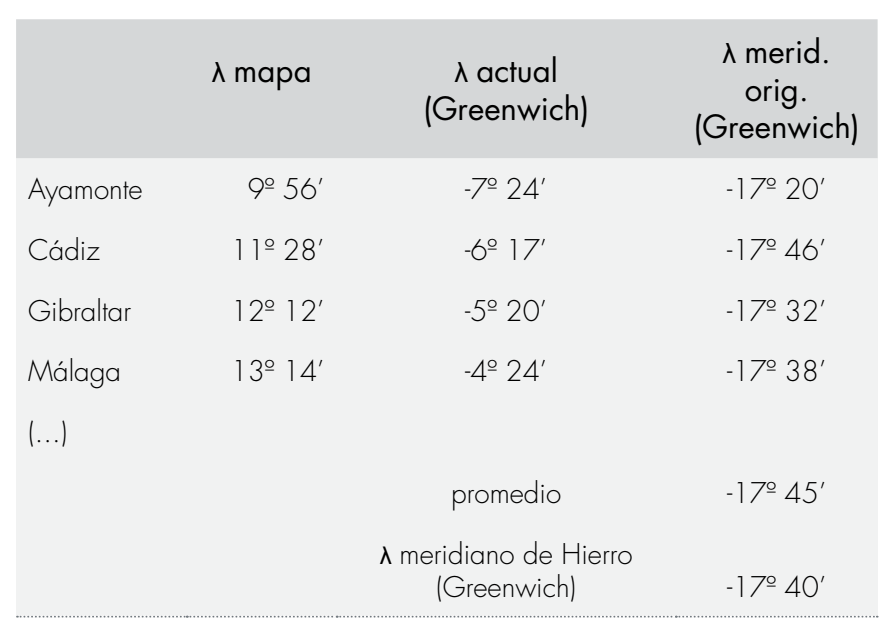

Tabla para el cálculo del meridiano origen (J. Bleau, 1636) 

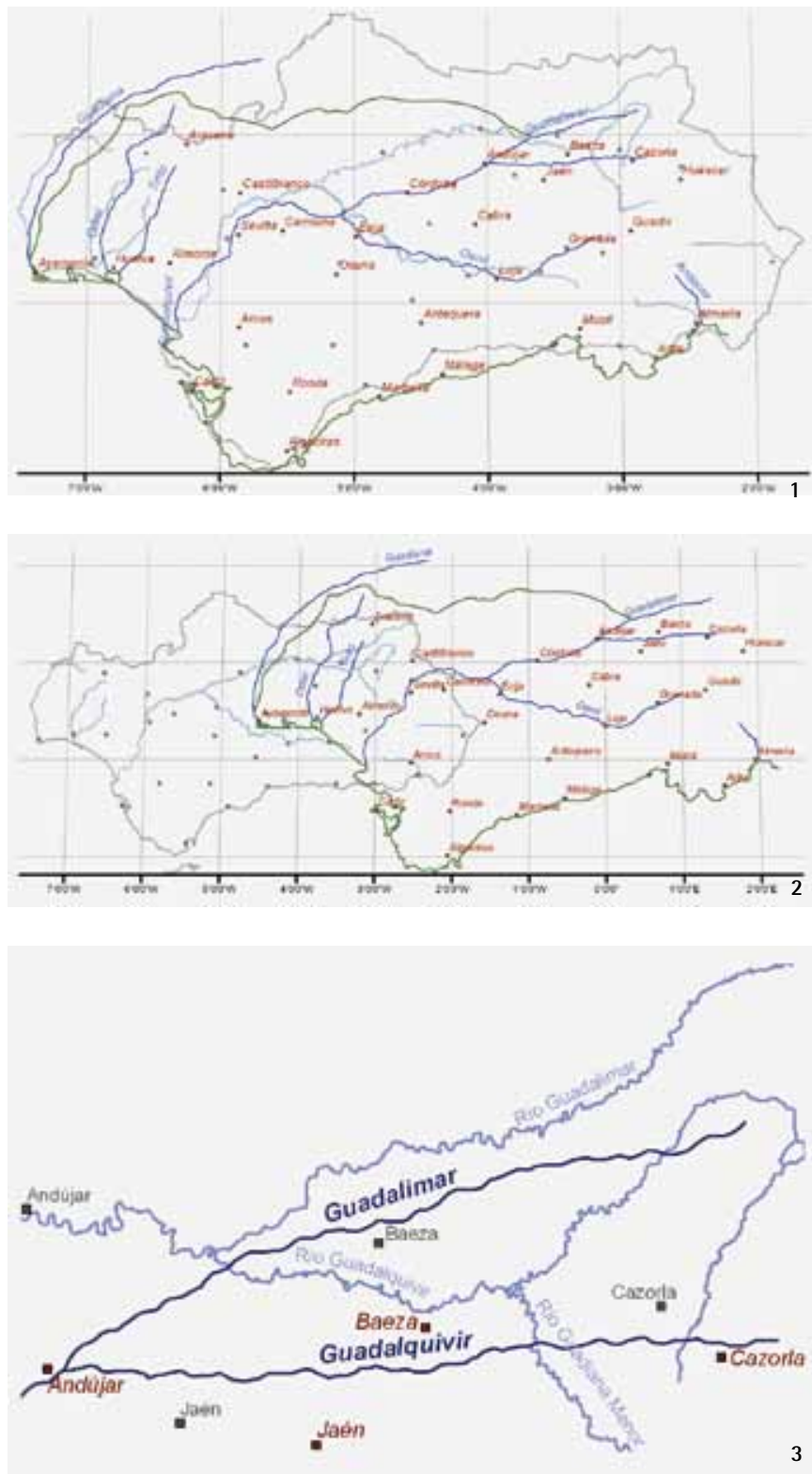

3

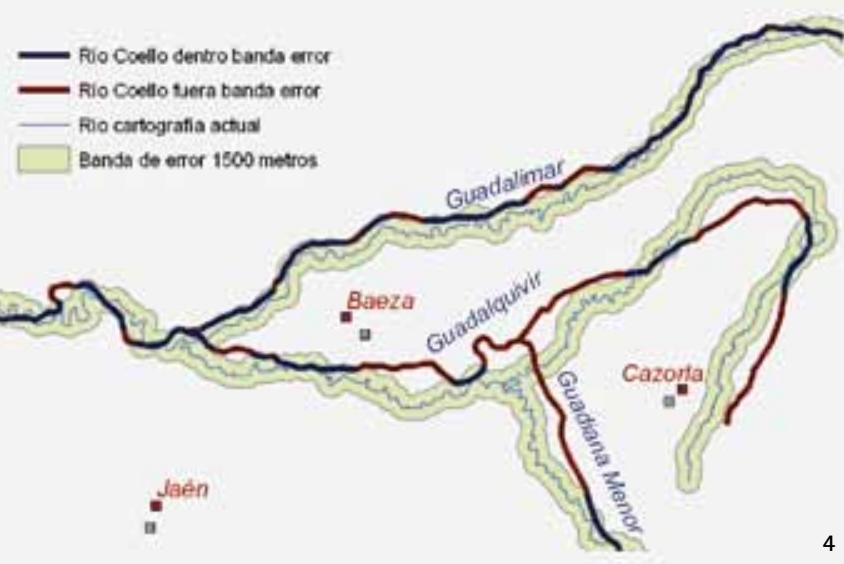

1. Capa vectorial georreferenciada en modo absoluto aplicando factor 1,31 a latitud/longitud (J. Hondius 1606) frente a cartografía actual.

Fuente: elaboración propia

2. Capa vectorial georreferenciada en modo absoluto (J. Hondius, 1606) frente a cartografía actual. Fuente: elaboración propia

3. Cabecera de ríos Guadalimar y Guadalquivir (J. Hondius, 1606) frente a cartografía actual. Véase la simplicidad del trazado.

Fuente: elaboración propia

4. Cabecera de ríos Guadalimar, Guadalquivir y Guadiana Menor (F. Coello, 1864) sobre banda de error de $1.500 \mathrm{~m}$ de la cartografía actual.

Fuente: elaboración propia có a todas las marcas de latitud/longitud de la carta el factor de escala 1,31. El resultado es muy ajustado a la realidad, por lo que puede apuntar a la posibilidad de que la estimación del radio de la Tierra en la época fuese inferior a la real (del orden de un 30\%).

\section{VECTORIZACIÓN}

Una vez georreferenciada la imagen del mapa, conviene vectorizar los elementos que se consideran de interés y que servirán de base para el análisis de la exactitud posicional. En el trabajo realizado se seleccionaron ciudades y ríos de relativa importancia que aparecen en casi todos los mapas estudiados con objeto de poder comparar la serie histórica. Estos elementos fueron vectorizados al igual que la línea de costa y el límite septentrional (cuando aparece). Indicar que las ciudades se han considerado como elementos puntuales debido a la escala de los mapas.

\section{EVALUACIÓN DE LA EXACTITUD}

La evaluación de la calidad posicional en la cartografía antigua se realiza aplicando análisis estadísticos de las discrepancias en las posiciones de los elementos similares a los que se utilizan en los controles de los datos geográficos actuales. No obstante, el análisis visual y el empleo de métodos gráficos toman relevancia ya que a menudo el interés se centra en observar el grado de similitud en las formas, las tendencias en los desplazamientos, etc., aspectos dificiles de cuantificar numéricamente, pero que destacan visualmente.

Un método gráfico muy ilustrativo consiste en trazar vectores uniendo la posición real de un punto con su homóloga en el mapa estudiado. El conjunto de vectores proporciona una visión de los errores posicionales y de las deformaciones presentes en la cartografía. Permite detectar sistematismos o tendencias y ayuda a extraer conclusiones sobre los errores que se podian estar cometiendo. Por otro lado, a partir de las coordenadas de las ciudades digitalizadas se pueden extraer las diferencias con sus posiciones reales y elaborar tablas y estadisticas de los errores. Si el mapa está georreferenciado en modo absoluto, estos valores serán una medida de la exactitud posicional absoluta de la cartografía.

También es interesante determinar la distancia y la orientación en el mapa entre las ciudades digitalizadas. La comparación de estos valores con los reales es una medida de la exactitud relativa de la cartografía. En el trabajo realizado, algunos de los errores extraídos se repiten en diferentes mapas y dan una idea de cómo se partía de información existente en la elaboración de los mapas y sistemáticamente se cometían equivocaciones similares.

Los elementos lineales, la costa y los ríos, son analizados normalmente de manera visual comparando las formas que presentan y los trazados; aunque también existen métodos de control que arro- 

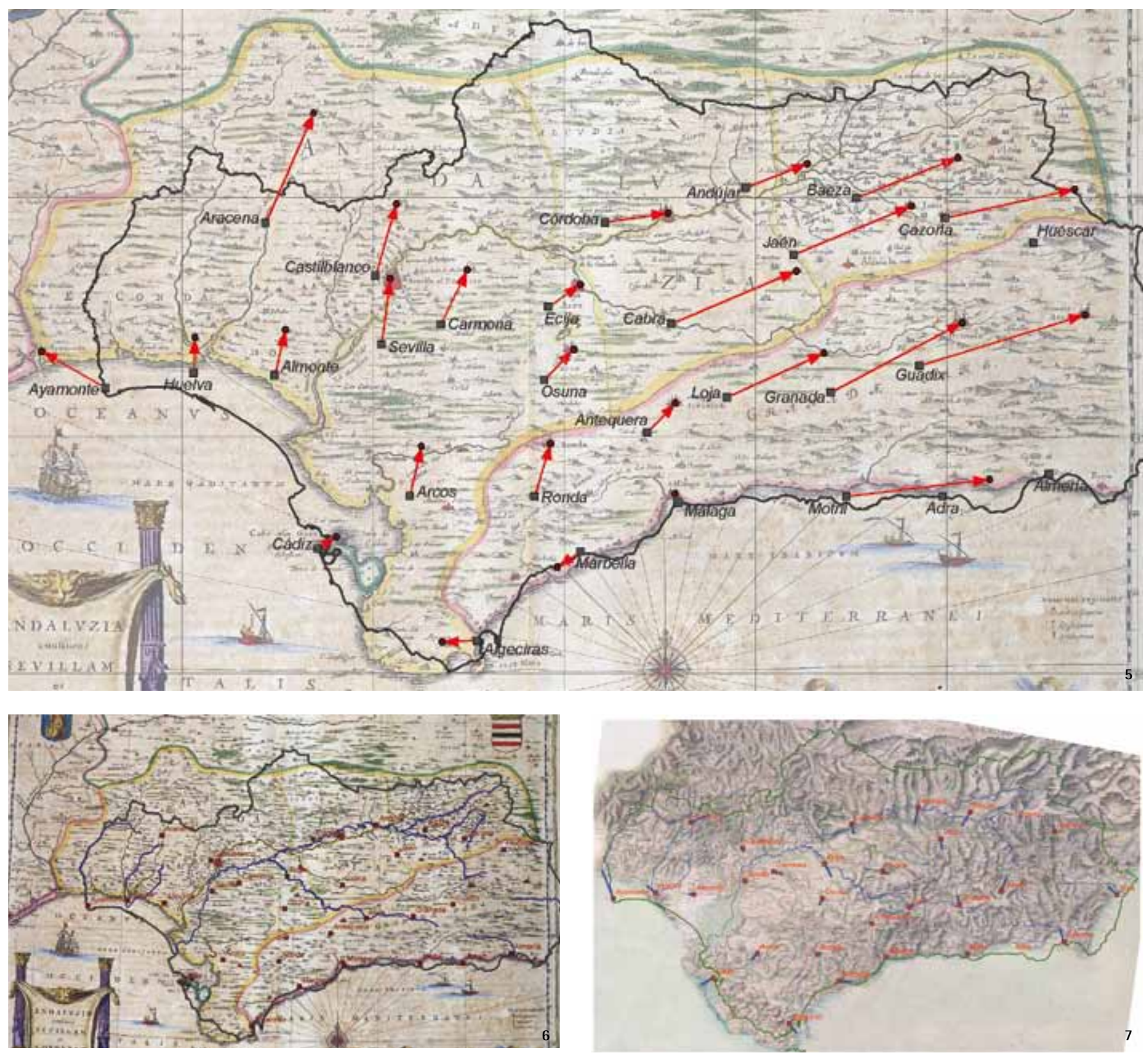

5. Vectores de error (J. Bleau 1636). Véase el fuerte desplazamiento hacia el este de la parte oriental. Fuente: ICA

6. Georreferenciación absoluta en Mercator y convertido a UTM huso 30 (J. Bleau, 1636). Cartografía actual como referencia. Fuente: ICA

7. Georreferenciación relativa en Cónica Conforme de Lambert (Mapa de los Jesuitas, 1739-1743). Las líneas azules muestran los residuos de los puntos utilizados.

Fuente: Real Sociedad Geográfica, depositada en la Biblioteca Nacional

jan resultados numéricos sobre su exactitud. Por ejemplo, se puede aplicar algún test basado en la banda de error que indique el grado de desplazamiento de la línea con respecto a la realidad.

No obstante, la conveniencia de aplicar un método numérico o simplemente hacer un análisis visual dependerá del tipo de mapa, ya que en ocasiones el trazado de las líneas responde a criterios estéticos y de legibilidad. Así, en los más antiguos, las formas de la costa suelen estar exageradas y los trazados de los ríos muy esquematizados. En ellos, el análisis visual permite extraer conclusiones sobre cómo se han cartografiado estos elementos, el nivel de rigor o de expresividad empleado.

En cambio, en los mapas más modernos, sobre todo del siglo XIX, la métrica cobra valor y los trazados tratan de ajustarse lo más po- sible a la realidad, con las limitaciones de la época. En estos casos tiene sentido la aplicación de algún método analítico que permita cuantificar el grado de desplazamiento de las líneas.

\section{CONCLUSIONES}

Como ha quedado expuesto, no resulta fácil establecer un método de trabajo fijo ya que la variedad de técnicas cartográficas y de errores que se pueden encontrar es grande, por lo que se han planteado una serie de directrices que se consideran adecuadas al abordar este tipo de estudios. Las decisiones tomadas en el análisis se han intentado siempre ilustrar con ejemplos concretos de mapas de Andalucia. El propósito no es dar un método definitivo sino animar a investigar este tipo de análisis para conocer y valorar con mayor profundidad la cartografía histórica. 